Musées, Patrimoine et Culture scientifiques et techniques

$123 \mid 2009$

mai - juin 2009

\title{
Initiatives européennes et patrimoine universitaire
}

Sébastien Soubiran, Marta C. Lourenço, Roland Wittje, Sofia Talas et Thomas Bremer

\section{OpenEdition Journals}

Édition électronique

URL : http://journals.openedition.org/ocim/229

DOI : 10.4000/ocim.229

ISSN : 2108-646X

Éditeur

OCIM

Édition imprimée

Date de publication : 1 mai 2009

Pagination : 5-14

ISSN : 0994-1908

Référence électronique

Sébastien Soubiran, Marta C. Lourenço, Roland Wittje, Sofia Talas et Thomas Bremer, « Initiatives européennes et patrimoine universitaire », La Lettre de I'OCIM [En ligne], 123 | 2009, mis en ligne le 01 mai 2011, consulté le 21 avril 2019. URL : http://journals.openedition.org/ocim/229 ; DOI : 10.4000/ ocim. 229 


\title{
Initiatives européennes et patrimoine universitaire
}

\author{
Sébastien Soubiran, Marta C. Lourenço, \\ Roland Wittje, Sofia Talas et Thomas Bremer *
}

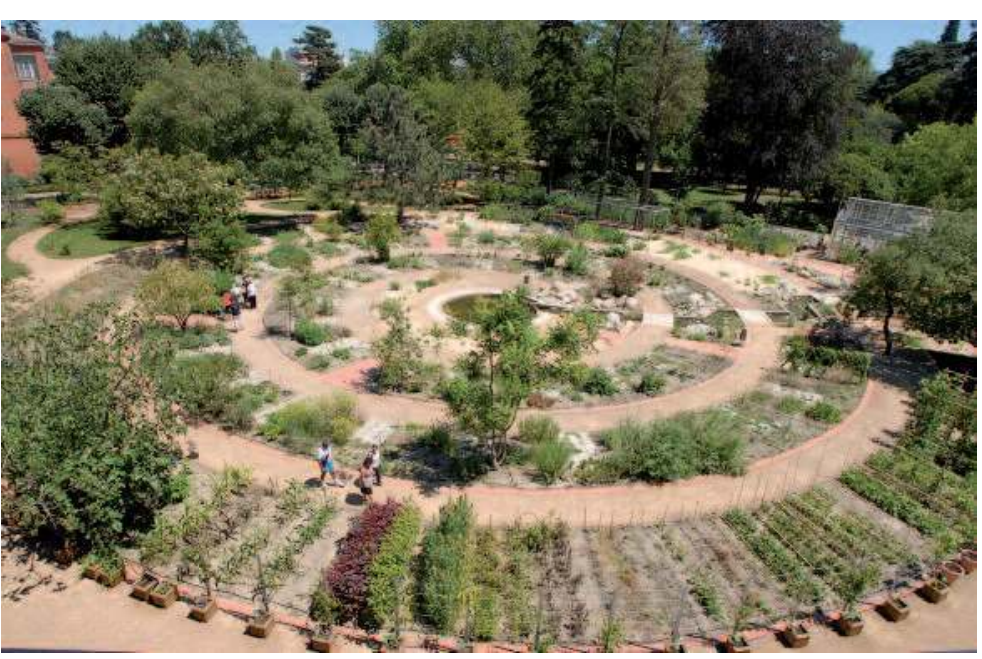

Le jardin botanique Henri Gaussen de l'université Paul Sabatier de Toulouse ๑) Muséum de Toulouse/Frédéric Ripoll

* Sébastien Soubiran est responsable des collections au jardin des Sciences, université de Strasbourg. Marta C. Lourenço est responsable des collections au musée de Sciences, université de Lisbonne. Roland Wittje est chercheur au département d'Histoire des Sciences, université de Regensburg. Sofia Talas est conservateur du musée d'Histoire de la Physique, université de Padoue.

Thomas Bremer est professeur à l'université de Halle.
Tout en effectuant un retour sur les actions de protection, de conservation, de valorisation et de diffusion du patrimoine scientifique universitaire menées depuis dix ans en Europe, des responsables de collections s'interrogent - à travers quatre exemples nationaux - sur la place de ce patrimoine au sein des universités et dans la société.

$\mathrm{Si}$, à l'échelle internationale, la grande fragilité du patrimoine des universités apparât encore comme un dénominateur commun, il reste indéniable que, durant les dix dernières années, l'intérêt pour les collections universitaires s'est considérablement renforcé. Un ensemble d'opérations témoigne d'un début de prise en compte par les puissances publiques et différents réseaux de professionnels (du patrimoine, des musées, du monde académique) de nombreux pays de questions relatives au patrimoine des universités, même si sur le terrain, les différents acteurs sont loin de rencontrer l'aide et les moyens nécessaires à des opérations d'envergure.

Cet article a pour objectif d'offrir un état des lieux de la situation du patrimoine universitaire qui complète celui qui avait été réalisé par Dominique Ferriot et Marta Lourenço il y a cinq ans (Ferriot et Lourenço, 2004). Il s'agit ainsi de prendre la mesure de ce qui a changé depuis. Plutôt qu'un état des lieux global à l'échelle internationale ou européenne, il nous a paru plus pertinent de revenir d'abord sur les initiatives les plus significatives au cours des dix dernières années et qui témoignent d'un renforcement des structures, 
de réseaux de professionnels, de projets dédiés au patrimoine des universités. L'organisation en réseaux de professionnels et la reconnaissance par les institutions politiques constituent en effet le fondement d'une action coordonnée autour de la préservation et la valorisation des collections et musées universitaires. Dans ce domaine, à l'échelle internationale, les trois initiatives les plus importantes sont certainement la création du réseau européen UNIVERSEUM en 2000, la fondation en 2001 d'un nouveau comité international dédié aux collections et musées universitaires (UMAC) au sein du Conseil international des musées (ICOM) et la rédaction par le Conseil de l'Europe de la Recommandation sur la gouvernance et la gestion $d u$ patrimoine universitaire (Conseil de l'Europe, 2004). Après une présentation de ces initiatives, nous examinerons la situation de quelques pays afin de rendre compte des disparités importantes d'un contexte national à l'autre, voire d'une université à une autre.

\section{Trois initiatives}

\section{UNIVERSEUM}

Le réseau européen de musées et collections universitaires, UNIVERSEUM, qui tire son nom des deux termes universitas et museum, a été créé en 2000 à l'initiative de l'université de Halle-Wittenberg. Celleci était alors engagée dans la préparation d'une importante exposition destinée à marquer les 500 ans d'existence de l'université de Wittenberg (2002). Cette préparation a permis une double prise de conscience. D'une part, qu'il existait une grande quantité de collections universitaires, relativement dispersées les unes par rapport aux autres et une grande diversité des états de conservation et des catalogages. Une série de discussions entamées entre Thomas Bremer, responsable politique du Rectorat de l'université de Halle-Wittenberg, Walter Tega (à l'époque vice-recteur à Bologne) et Liba Taub (directrice du Whipple Museum of the History of Science à Cambridge), ont conduit à faire le constat que de nombreux problèmes structuraux des musées universitaires en Europe étaient identiques. La liste de ces problèmes est malheureusement bien connue : c'est d'abord l'accès difficile pour un large public intéressé, c'est également l'état souvent précaire des spécimens, l'absence d'une documentation numérisée et d’informations électroniques sur les collections ainsi que sur leurs catalogues, mais c'est aussi l'état instable et souvent précaire du personnel «de musée » dans les restructurations politiques des universités en Europe...

La première action d'UNIVERSEUM est la «Déclaration de Halle» (avril 2000), qui a été le

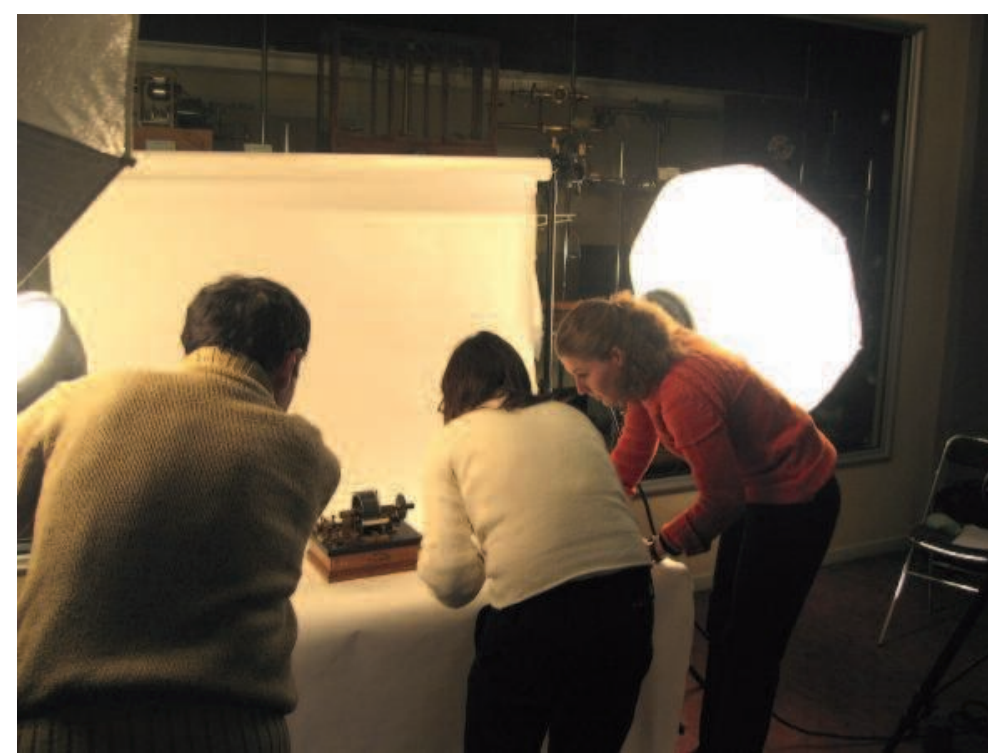

Travail d'inventaire des instruments de Physique à l'université de Strasbourg () Université de Strasbourg/S. Soubiran

résultat d'une conférence de 12 des universités européennes les plus anciennes, toutes en possession d'importantes collections, qui étaient dans certains cas conservées dans des musées universitaires. Cette déclaration essayait pour la première fois de formuler des exigences - auprès des universités, des gouvernements nationaux et des instances européennes - pour la définition d'une politique visant à améliorer la conservation et l'accès public du patrimoine académique de chacune des universités présentes. À côté de cet acte politique, un projet commun a matérialisé cette collaboration avec la publication - financée par la Commission Européenne dans le cadre du programme «Culture 2000 » et publiée en 2001 - d'un ouvrage collectif qui montrait la richesse des collections universitaires à l'échelle européenne. Ces actions ont rencontré plusieurs échos auprès des instances européennes : d'une part, la Commission Européenne a affirmé son intérêt en créant une « Journée Européenne des musées et collections académiques » (18 novembre) et d'autre part, le Conseil de l'Europe a débattu de la situation des collections et publié, en 2002, un certain nombre de recommandations (Sanz et Bergan, 2002).

Depuis UNIVERSEUM a approfondi sa mission, en s’intéressant à la dimension européenne du patrimoine des universités dans son ensemble - non seulement les musées et les collections, mais également le patrimoine immobilier, les archives et les bibliothèques. UNIVERSEUM a grandi au cours des 
années mais demeure un réseau libre sans membres fixes, sans statut, ni cotisation : les réunions annuelles instaurées dès $2000^{(1)}$ permettent ainsi à des représentants d'une centaine de collections universitaires, provenant d'une vingtaine de pays européens, de se rencontrer pour envisager des initiatives communes en faveur de la conservation et de la valorisation du patrimoine universitaire. Le site Internet, restructuré en 2008, contribue à améliorer en interne le contact entre les différents membres tout en renforçant la visibilité à l'extérieur des différentes actions du réseau ${ }^{(2)}$.

\section{UMAC (ICOM)}

Le Comité international pour les musées et collections universitaires (UMAC) a été formellement créé au cours de la $19^{e}$ assemblée générale du Conseil international des musées (ICOM) à Barcelone en juillet 2001. UMAC est le premier réseau de musées et collections universitaires d'envergure internationale. Sa création témoigne de la reconnaissance d'une identité distincte et officielle des musées universitaires en tant que «musées » par la plus importante organisation mondiale des musées. Selon Peter Stanbury, le premier président d'UMAC (2001-2004), "le rôle d'UMAC est de souligner les similarités et les différences entre les musées universitaires et les autres musées, et d'encourager l'interaction et la collaboration entre l'ensemble des professionnels des musées [...]. En posant des questions de fond, l'UMAC permet d'amener des solutions pour protéger notre patrimoine commun. Les publications, conférences et discussions d'UMAC augmentent la formation formelle des responsables des collections universitaires »(Stanbury, 2003).

L'UMAC s'est montré très actif depuis sa création. Tout d'abord, il s'est engagé dans une politique éditoriale visant à mettre à disposition des travaux et des ressources documentaires qui faisaient souvent défaut. Ainsi, ont été publiés tous les actes de ses conférences annuelles (3), une compilation d'un ensemble de textes et l'édition d'un numéro de l'ICOM Study Series (ICOM, 2003) ainsi qu'un document de travail intitulé University Museums and Collections: Importance, Responsibility, Maintenance, Disposal and Closure (UMAC, 2004). L'UMAC s'est également engagé dans la mise en place d'une base de données qui réunit des informations sur les musées et collections universitaires du monde entier. Cette base de données, en cours d'enrichissement, sera un outil de travail utile pour les professionnels de musées, les chercheurs et le grand public (Weber et Lourenço, 2005) (4).
Les recommandations du Conseil de l'Europe

Une autre initiative importante concernant le patrimoine universitaire a été prise à Strasbourg le 7 décembre 2005, lorsque le Conseil de l'Europe a adopté à l'unanimité une recommandation relative au patrimoine universitaire ${ }^{(5)}$. Bien qu'ayant eu jusquà maintenant un impact limité, cette recommandation Rec(2005)13, intitulée Recommandation sur la gouvernance et la gestion du patrimoine universitaire, est le résultat d'une collaboration entre deux comités et services différents du Conseil - l'un chargé du patrimoine culturel et l'autre de l'enseignement supérieur et de la recherche. Elle s'adresse en premier lieu aux gouvernements des états membres et aux directions des universités. Ce document contient des recommandations spécifiques concernant les politiques, la législation, la gouvernance et la gestion, le financement, l'accessibilité, la formation professionnelle, la recherche, la sensibilisation, les relations avec les collectivités territoriales, et la coopération internationale. La recommandation encourage les directions des universités «à considérer l'ensemble du patrimoine de l'établissement d'enseignement supérieur comme relevant de [leur] responsabilité ultime, morale, administrative et juridique » et demande aux autorités de tutelle et aux universités de " prévoir le financement de leur politique du patrimoine dans leur propre budget, qu'il soit alimenté par des fonds publics ou privés, et de chercher à obtenir des ressources supplémentaires à l'extérieur».

\section{La situation dans 4 pays européens}

\section{Les musées et collections universitaires en Allemagne}

La situation des collections et musées universitaires en Allemagne est assez hétérogène. Elle résulte, d'une part, de l'organisation fédérale de l'Allemagne, qui place les universités sous la responsabilité de 16 États Fédéraux, et d'autre part, de l'importante autonomie qui caractérise, aujourd'hui encore, les universités et leurs départements. Les collections appartiennent généralement aux départements universitaires, qui manquent souvent de connaissances et de savoir-faire en matière de conservation muséale et d'espaces appropriés pour le stockage des collections. Les collections sont dans la plupart des cas insuffisamment documentées et mal inventoriées et protégées. Comme dans d'autres pays, le manque de financements et de reconnaissance semblent être de règle, et la préservation des collections, qui dépend dans la plupart des cas d'individus, peut être menacée lors d'un départ à la retraite ou d'une restructuration du département. 


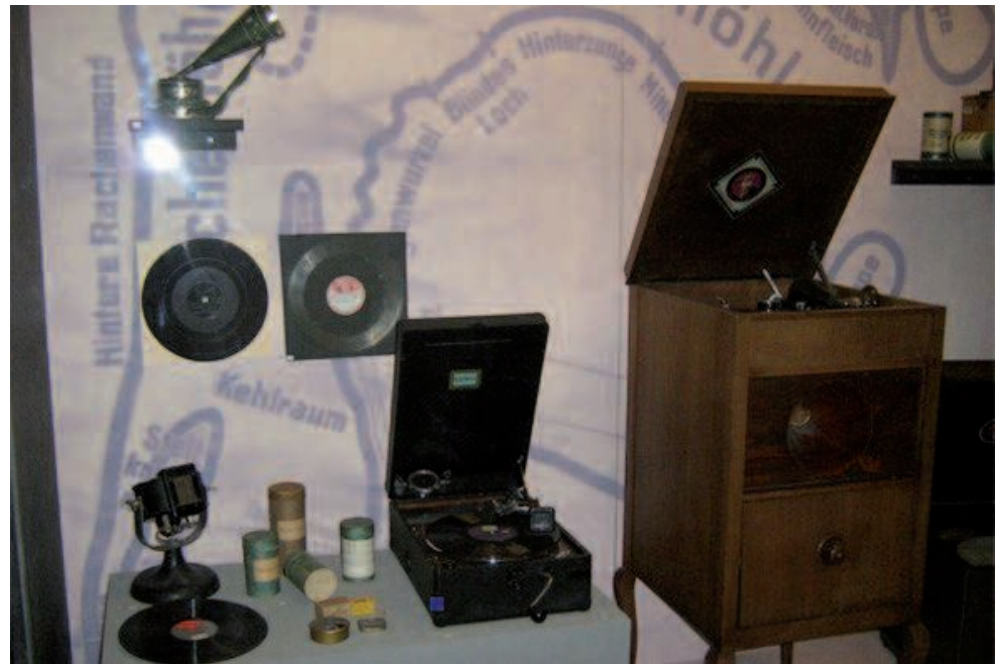

Collections d'instruments d'acoustique de l'université de Halle-Wittenberg (c) Université de Halle-Wittenberg

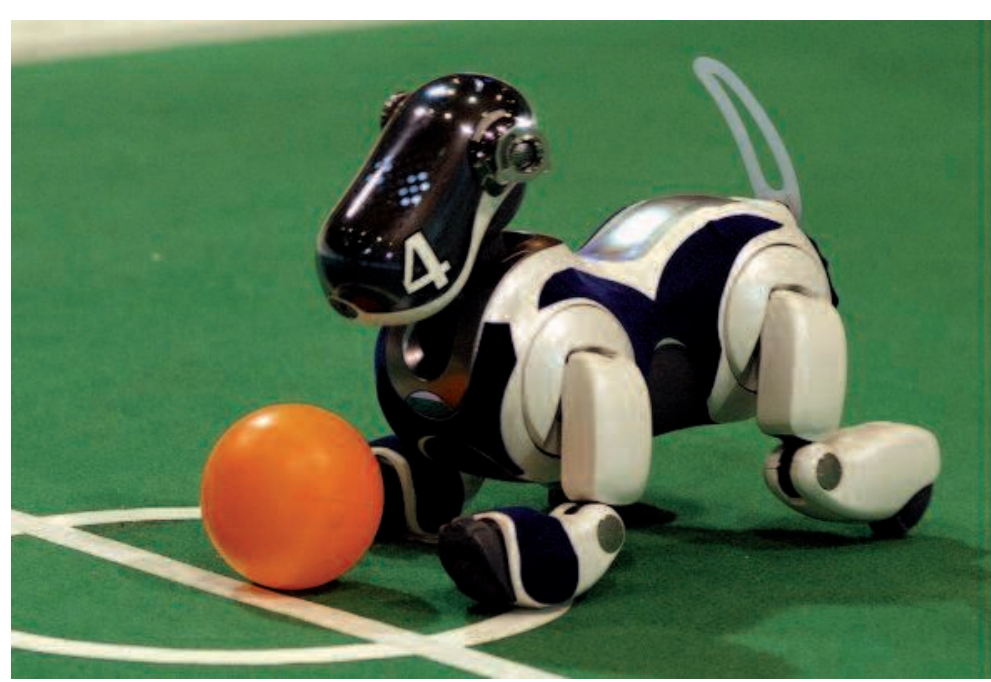

Ce chien robot AIBO (Artificial Intelligence Robot) ERS-7, développé en 1999 par Sony, est issu des collections scientifiques de l'université Humboldt, Berlin. (C) Université Humboldt

Dans les universités de l'ex-Allemagne de l'Est, une institution centrale chargée de la conservation (Kustodie) avait été créée dans chaque université afin de préserver les collections universitaires traditionnelles et les collections d'art. À la TU Bergakademie Freiberg et à l'université technique de Dresde, la Kustodie comprend également les collections de science et d'ingénierie ${ }^{(7)}$. Une telle institution centrale fait défaut dans la plupart des universités de l'ancienne Allemagne de l'Ouest, notamment dans les universités de Göttingen et de Heidelberg qui possèdent chacune de nombreuses collections d'une grande valeur ${ }^{(8)}$.

Enfin, parmi les projets les plus récents et intéressants, il faut mentionner la mise en chantier du musée de l'université de Tübingen démarré en 2006 et qui lie les collections à des programmes de recherche en Histoire des Sciences et associe des artistes ${ }^{(9)}$.

\section{Les musées et collections universitaires en Italie}

Liées au morcellement politique qui a caractérisé l'Italie jusqu'à une époque récente, richesse et fragmentation semblent être les principales caractéristiques du patrimoine universitaire italien. L'étude conduite en 2002 par la CRUI (association des universités Italiennes qui regroupe les Recteurs de toutes les universités du pays) a ainsi compté 359 collections, dont 178 musées, réparties sur 32 universités de l'ensemble du territoire national (10). Cette étude de la CRUI a été conduite dans le cadre d'un 
accord de financement entre la CRUI et le ministère de l'Université et de la Recherche scientifique et technologique en vue de la réalisation d'un projet de réseau national des musées universitaires (responsable du projet : Giulio Peruzzi, université de Padoue). Toutefois, peu d'efforts ont étés mis en œuvre à ce jour pour offrir une gestion coordonnée au niveau national. La situation actuelle des musées universitaires en Italie est dans l'ensemble critique et se caractérise par des différences très marquées d'une université à l'autre.

La CRUI avait pourtant mis en place dès 1999 une "Commission pour les musées, les archives, les collections universitaires » afin de "relancer l'important patrimoine de notre Université et de promouvoir la coordination entre les réalités académiques » (11). Un des résultats concrets les plus significatifs obtenus par la Commission a été la réalisation en 2002 de l'étude présentée plus haut. Pour la première fois en Italie, celle-ci a conduit à une analyse précieuse, systématique et complète, des différents paramètres qui caractérisent les collections et musées universitaires du territoire (ressources humaines, statuts et règlements, financements, surface, ouverture et accès, services, bases de données). Le rapport proposait en outre le projet d'un réseau national des musées universitaires mais, à la suite de changements dans la composition de l'assemblée des recteurs de la CRUI, ce projet n'a connu jusqu'à maintenant aucun développement concret.

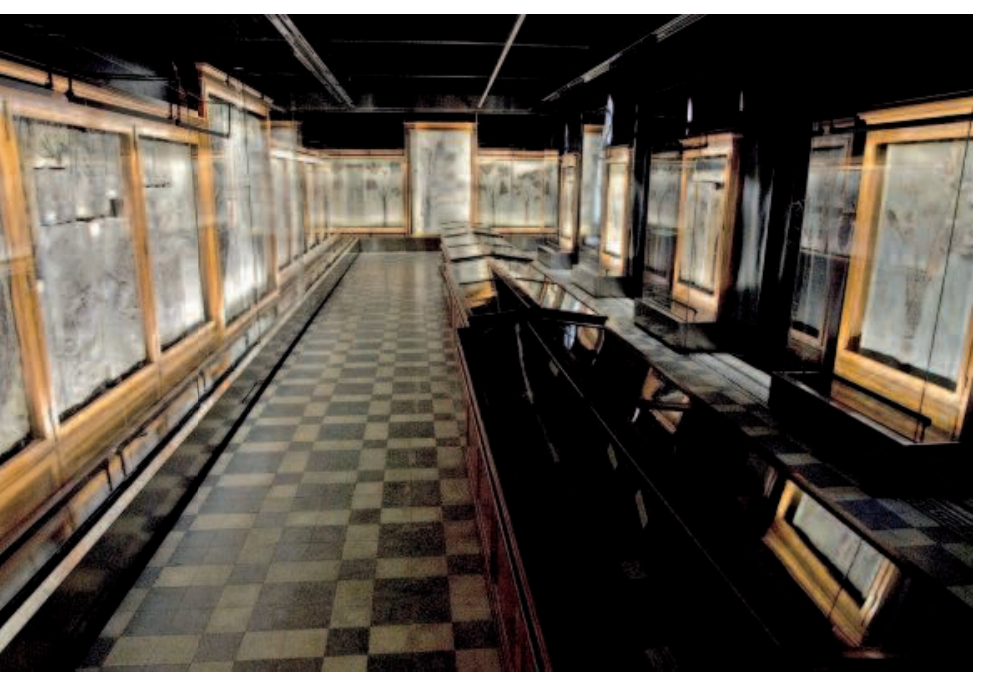

La Salle des Palmes du musée de Géologie et Paléontologie de l'université de Padoue (c) Université de Padoue
Les réunions de la Commission pour les musées de la CRUI se sont en fait de plus en plus espacées au cours de ces dernières années et l'activité de la Commission apparaît aujourd'hui pratiquement éteinte. Il faut cependant souligner qu'à travers la Commission pour les musées, la CRUI a été l'un des trois partenaires d'un accord établi en mars 2005 entre l'Institut Central pour le Catalogage et la Documentation (ICCD) du ministère pour les Biens Culturels et l'ENEA (Organisation pour les nouvelles technologies, l'Energie et l'Environnement). Il s'agissait d'un accord pour «la recherche, les études et la formation dans le secteur du catalogage ». Des groupes de travail ont été créés pour établir des normes nationales de catalogage dans différents secteurs (patrimoine scientifique et technologique, botanique, minéralogie, zoologie, anthropologie, paléontologie...). Dans le cadre de ces groupes de travail, les conservateurs ou chercheurs des universités ont souvent joué un rôle crucial. En ce qui concerne par exemple les critères de catalogage adoptés pour le patrimoine paléontologique, les propositions des conservateurs du musée de Paléontologie de l'université de Padoue ont été intégralement retenues et constituent donc désormais le standard national pour le catalogage de ce type de patrimoine.

De fait, il s'agit là du seul cadre dans lequel une réelle coordination a été mise en œuvre. Pour le reste, chaque université affronte de façon autonome la gestion de ses propres collections, avec des situations de négligence grave dans certains cas et des musées organisés en réseaux très actifs dans d'autres cas : les universités de Bari, Bologne, Cagliari, Florence, Milan, Naples, Padoue, Rome et Turin, par exemple, ont les activités de leurs musées coordonnées par des Centres ou des «Sistemi museali ». Ce qui n'empêche pas d'avoir, au sein d'une même université des situations d'excellence côtoyant des situations catastrophiques. À Bologne par exemple, une partie des collections est magnifiquement exposée et mise en valeur au Palazzo Poggi, tandis que les collections du musée d'Histoire de la Physique, conservées il y a encore quelques années dans le département de Physique, sont actuellement stockées dans un dépôt inaccessible au public.

Enfin, l'un des problèmes certainement les plus graves pour les musées universitaires en Italie demeure celui du personnel. Dans la plupart des cas, ce sont des chercheurs ou des professeurs qui s'occupent des collections sans aucun caractère officiel : ces collections risquent bien sûr de sombrer dans l'oubli avec le départ à la retraite ou le déplacement des 


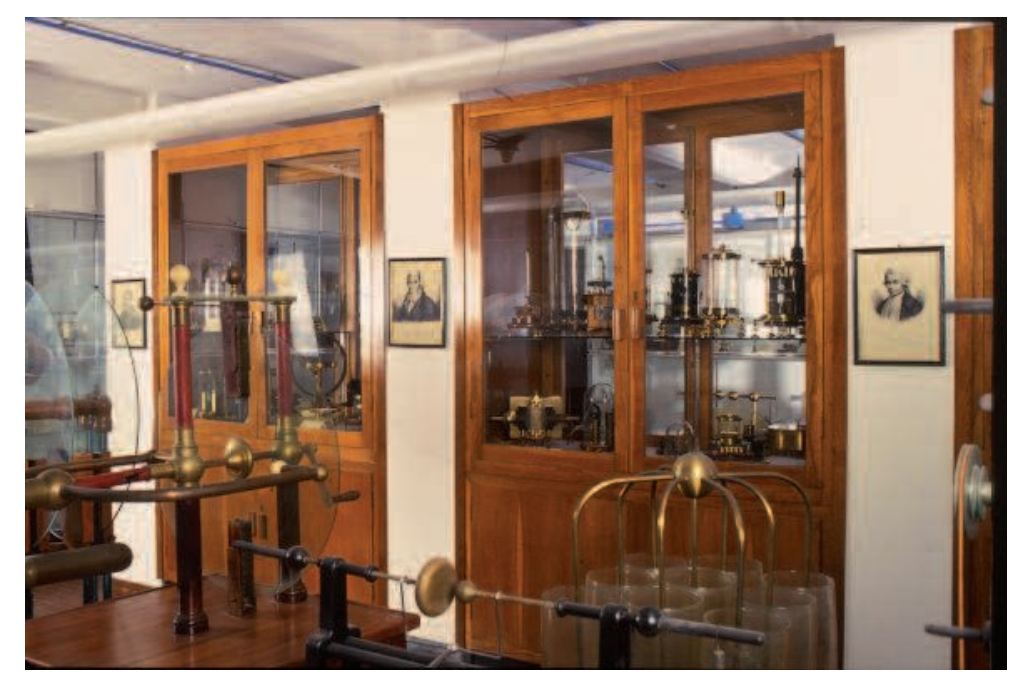

Section sur l'électricité au musée d'Histoire de la Physique, université de Padoue (c) Université de Padoue

personnes qui s'y intéressent. Dans certains cas par contre, des postes de conservateurs spécifiques ont été créés mais, en 2002, l'on ne comptait encore que 90 conservateurs pour les 178 musées recensés et, pour l'instant, les universités ne semblent pas encore prêtes à envisager un investissement sérieux dans ce domaine. Il est significatif par exemple que la "Carta nazionale delle professioni museali ", élaborée en octobre 2005 par l'ICOM Italie et par l'association des musées italiens, n'ait pas été ratifiée par la CRUI, qui s'est justifiée en donnant pour motif les «spécificités des musées universitaires ». Un long chemin reste donc à parcourir pour une valorisation sérieuse du patrimoine universitaire italien.

\section{Les musées et collections universitaires en France}

Un regard rapide sur les modes de gestion et de valorisation des collections universitaires mis en œuvre en France, fait apparaître une grande hétérogénéité des réponses apportées, liées bien sûr à la grande variété des collections mais également à leur mobilisation ou non dans une dynamique de recherche ou d'enseignement. La principale difficulté, comme dans d'autres pays européens, consiste en la qualification et la reconnaissance d'une valeur patrimoniale pour ces collections. Le patrimoine des universités est toutefois l'objet d'une préoccupation croissante en France depuis les dix dernières années (12), suivant en cela la dynamique générale à l'échelle européenne (Boudia, 2007). Celle-ci se traduit, en même temps qu'elle est renforcée, par la création de nouvelles structures (13), l'émergence de nouveaux réseaux sur le plan national, composés d'acteurs du monde académique, des musées et du patrimoine, mais aussi parfois des représentants d'administration de l'État. Ces réseaux peuvent être structurés autour d'objectifs précis tels que la préservation du patrimoine scientifique contemporain ${ }^{(14)}$, l'inventaire national des types et figurés paléontologiques (15), ou plus informels, suite à l'organisation de colloques, conférences, symposium (à Lille en 2004, Montpellier en 2005, Nancy en 2006 et Strasbourg en 2009). Depuis l'année 2008, un nouveau pas a été franchi avec la création d'une nouvelle branche d'activité professionnelle au sein des personnels de l'université, en lien avec la gestion des collections universitaires, (assistant, chargé et responsable de collections muséales). Cette reconnaissance dans le champ patrimonial et muséal entre autres, s'accompagne d'une part d'actions sur le terrain visant à sa préservation, sa mise en public et d'autre part de travaux académiques cherchant à mieux les comprendre, les décrire, en établir une typologie ; l'ensemble contribuant à développer notre connaissance de ce que le patrimoine des universités recouvre (Université des Sciences et Technologies de Lille, 2004 ; Gérard, 2008 ; Université de Strasbourg, 2009 ; Boudia, Rasmussen et Soubiran, à paraître).

Entre autres caractéristiques dans le paysage français, soulignons l'imbrication marquée entre des structures centralisées (lorsqu'elles existent) au sein d'une université en charge des musées et collections ou d'une activité patrimoniale, avec celles chargées des actions de diffusion de la culture scientifique et technique (ce sont souvent les mêmes structures). La valorisation du patrimoine participe ainsi aux enjeux de diffusion et de promotion des sciences et des carrières scientifiques qui sous-tendent les activités de diffusion de la culture scientifique et technique en France (Bergeron, à paraître). Ce développement commun n'est pas sans ambiguïté, la culture scientifique et technique tendant à se démarquer du patrimoine pour promouvoir une science innovante, attractive, dynamique. En outre, la préservation du patrimoine scientifique et son accessibilité au public s'inscrit rarement dans une politique pérenne, avec ce qu'elle impose comme règles dans son administration, dans sa mise en public, ses savoir-faire et son encadrement professionnel. La préservation du patrimoine doit encore trouver une légitimité propre, voire un sens, au sein des universités en France. 


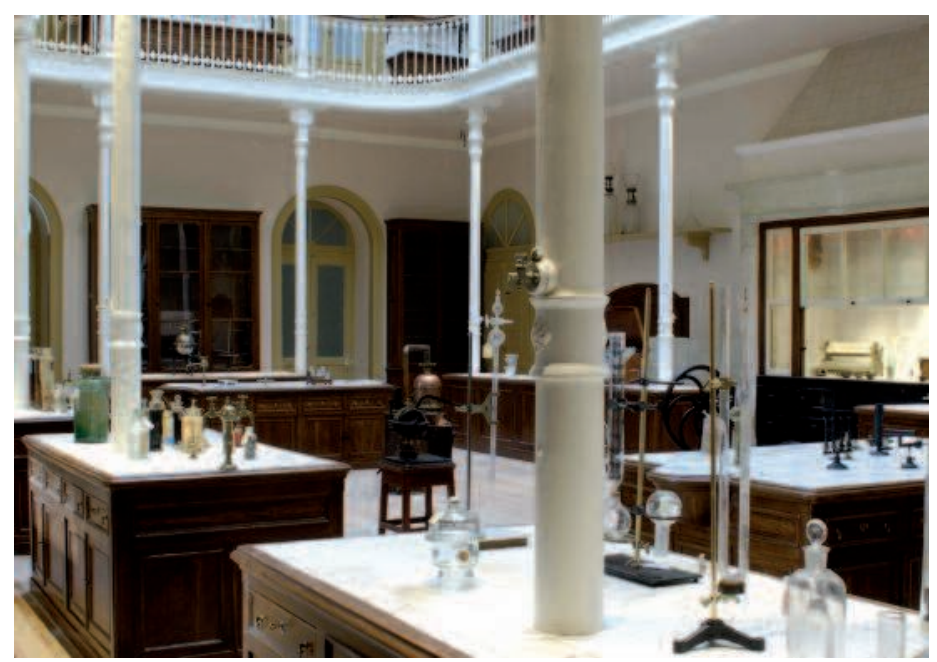

Laboratoire de Chimie de l'École polytechnique, musée de Science de l'université de Lisbonne (XIXe siècle) ๑) Musée de Science/P. Cintra

ce patrimoine est constitué de collections d'art et de sciences qui dans leur majorité ne sont pas préservées dans un musée (quand elles le sont, ces musées ne sont pas régulièrement ouverts au public). Il existe ainsi environ 40 à 50 musées et collections conservés dans les 12 universités publiques du pays (17). Aucune enquête nationale n’a été menée sur l'ensemble des musées et collections mais on peut estimer que le patrimoine scientifique des universités représente probablement $80 \%$ de l'ensemble du patrimoine scientifique du Portugal. Le musée national d'Histoire naturelle est ainsi sous la tutelle de l'université de Lisbonne. Limportance du cabinet de Physique de l'université de Coimbra (XVIIIe siècle), les trois laboratoires de Chimie des universités de Coimbra (XVIII ${ }^{\mathrm{e}}$ siècle), de Lisbonne (XIXe siècle) et de Porto (XXe siècle), et les deux observatoires astronomiques (XIXe siècle) de l'université de Lisbonne, dépassent les frontières nationales par leur rareté et leur singularité. D’une façon générale, ce patrimoine manque de financements et d'encadrement institutionnel, mais plus important encore, il manque de visibilité publique, d'identité et d'une mission bien définie.

L'université qui est clairement la plus en avance pour l'organisation et la reconnaissance d'un statut institutionnel de ses musées et collections est l'université de Coimbra qui, au début des années 2000, s'est engagée dans une réforme structurelle visant à fusionner les musées et les collections de la faculté des Sciences et Technologies (incluant le cabinet de Physique et de Chimie mentionnés plus haut mais

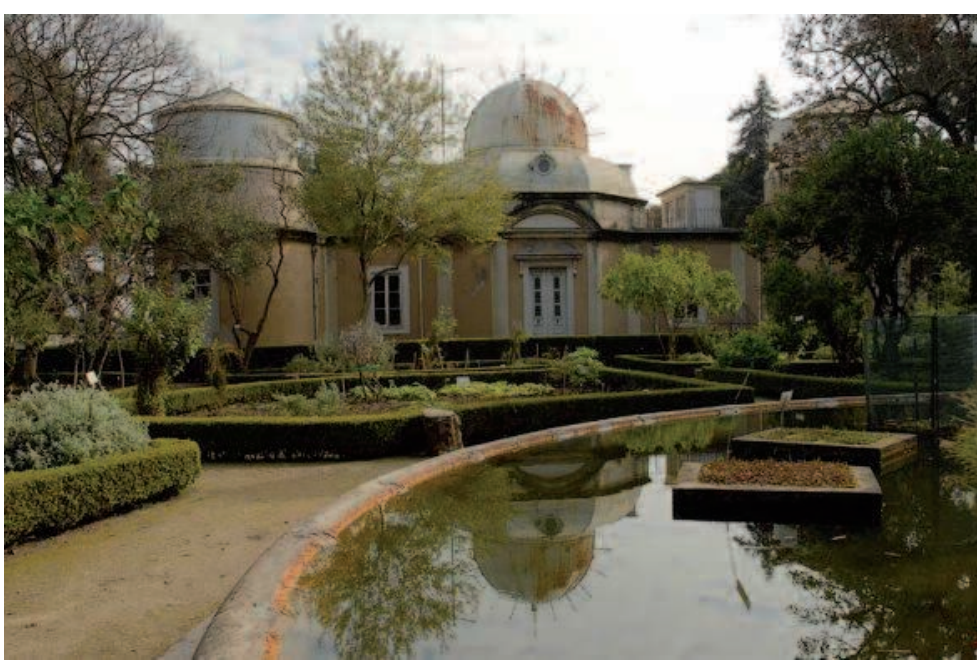

Observatoire astronomique de l'École Polytechnique, musée de Science de l'université de Lisbonne : vue de la façade arrière du pavillon reconstruit en 1898, avec au premier plan l'un des bassins du jardin botanique ๑ Inventaire général/M. Heller

également les collections d'Astronomie et d'Histoire naturelle). Cette fusion a abouti à la création du musée des Sciences de l'université de Coimbra (MOTA), dont la préfiguration a été inaugurée en mai 2006 et qui a mis en ligne ses collections depuis 2008, date à laquelle il s'est vu décerner le "Prix Micheletti, Prix Européen du Musée de l'Année », catégorie Science et Technologie (18).

Parallèlement, ces dernières années ont vu une augmentation significative des études consacrées aux collections universitaires portugaises (Lourenço, 2007 ; Garradas, 2008). Ces études ont été grandement stimulées par les conférences internationales organisées récemment à Lisbonne (UNIVERSEUM en 2007, The Scientific Instrument Commission en 2008) et plusieurs thèses de troisième cycle sont ainsi en cours. Même si un effort reste à faire en terme de visibilité internationale, on peut donc espérer que ces travaux permettront de soutenir des prises de décision à la fois par l'administration des universités et par le gouvernement. Enfin, l'une des plus importantes agences gouvernementales de la recherche - la Fondation pour la Science et la Technologie - a décidé de financer une enquête nationale sur le patrimoine scientifique du Portugal, qui comprendra bien sûr le patrimoine universitaire : cette enquête devrait démarrer au cours de l'année 2009 sous la direction des universités de Lisbonne, Coimbra et Porto. 


\section{Conclusion : une grande fragilité et des défis considérables}

Depuis la Deuxième Guerre mondiale, les musées et collections des universités ont connu une diminution de leur utilisation dans la recherche et l'enseignement avec pour conséquences un manque de ressources financières, d'autonomie, d'accessibilité et de locaux adéquats, un sous-équipement chronique en personnel qualifié, des réorganisations, des dispersions, des déménagements, des états de négligence et d'abandon, qui ont entraîné des pertes irréversibles. En même temps, en Europe, les systèmes d'enseignement supérieur connaissent aujourd'hui des transformations radicales et une crise profonde face à des demandes sociales et politiques parfois contradictoires. Cette situation a accentué la vulnérabilité

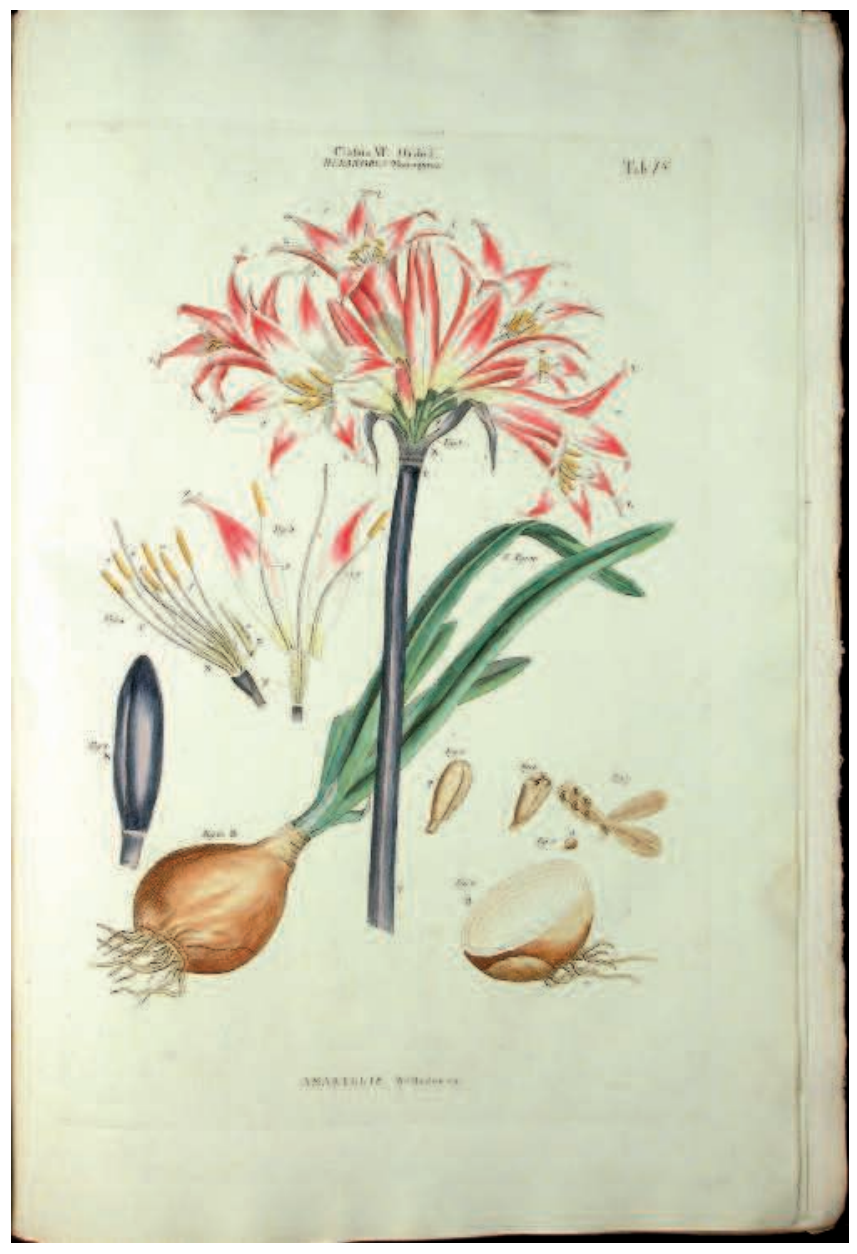

Amarillis Belladonna, gravure sur cuivre de Johann Sebastian Müller publiée dans Illustratio Systematis Sexualis Linnaei (108 p., traduction allemande de 1804), ouvrage appartenant au fonds de livres anciens de la bibliothèque de l'université Humboldt. (๖) Université Humboldt des musées et collections universitaires en Europe et impose le devoir d'agir avec encore plus d'urgence. Au vu des profonds remaniements en cours au sein des universités européennes, engagées dans le processus de Bologne, ce qui peut paraître acquis aujourd'hui est susceptible de renégociations demain et entrainer de nouvelles pertes. Cette instabilité fait partie du quotidien de nombreux petits musées universitaires, de collections, d'archives, de jardins botaniques dans toute l'Europe (19). Quand ceux-ci disparaissent, comme cela est déjà arrivé dans un grand nombre de cas, peu de personnes s'en aperçoivent - près de $80 \%$ du patrimoine universitaire prenant une forme non reconnue par les musées, les instances culturelles ou le public ${ }^{(20)}$.

Plus que jamais le patrimoine des universités doit donc faire face à de nombreux défis. Certaines questions relatives à l'identité, l'histoire et le rôle du patrimoine universitaire demandent davantage de recherches et de publications. Il existe des questions d'ordre politique et institutionnel qui méritent un plaidoyer constant. Il existe des questions de visibilité qui nécessitent des actions de sensibilisation auprès de la société et des autorités culturelles. Il existe des questions de préservation qui demandent davantage de formation et d'échange. Et il y a également des questions financières qui nécessitent une attention particulière de la part des gouvernements. Toutes ces questions seront discutées en juin à l'université Paul Sabatier de Toulouse, dans le cadre de la 10e conférence annuelle d'UNIVERSEUM ${ }^{(21)}$. En outre, il est envisagé de consolider la structure interne d'UNIVERSEUM pour l'inscrire dans une perspective à long terme afin de fournir une plateforme adéquate pour la préservation, l'étude et la reconnaissance du patrimoine universitaire européen.

\section{Notes}

(1) Halle (2000), Utrecht et Berlin (2001), Bologne (2002), Cambridge (2003), Wittenberg (2004), Tartu (2005), Strasbourg (2006), Lisbonne (2007), Cracovie (2008), Toulouse (2009), Uppsala (2010, à confirmer). (2) Voir www.universeum.it

(3) En 2008, UMAC a créé une publication numérique pour la publication de ses conférences annuelles, UMAC Journal. Voir http://edoc.huberlin.de/umacj/

(4) Voir http://publicus.culture.hu-berlin.de/collections/

(5) L'intégralité du texte de la recommandation est accessible en ligne en français à l'adresse : http://wcd.coe.int/

(6) L'adresse du nouveau site Internet du Hermann von Helmholtz Zentrum für Kulturtechnik est : www2.hu-berlin.de/kulturtechnik ; les 
collections de l'université de Humboldt sont accessibles à l'adresse : www.sammlungen.hu-berlin.de/

(7) Pour les collections de Dresden voir :

http://tudresden.de/die_tu_dresden/zentrale_einrichtungen/kustodie.

(8) Voir www.uni-goettingen.de/de/419.html et www.uni-heidelberg.de/ univ/museen/

(9) Voir www.unimuseum.uni-tuebingen.de/

(10) Comme un peu partout en Europe, la différence entre musée et collection universitaires en Italie n'est pas toujours très nette. En général, les collections universitaires italiennes sont considérées comme « musées » si elles ont un espace dédié et si elles peuvent être visitées par le public, même de façon très limitée.

(11) Voir www.crui.it/HomePage.aspx?ref=891

(12) Dernier exemple d'intérêt accru, la rénovation du musée Fragonard (la Lettre de l'OCIM n 121 janvier-février 2009) ou le musée d'Éthnographie de Bordeaux 2 (www.meb.u-bordeaux2.fr/).

(13) Par exemple, le service des Collections, université de Montpellier 2 ; le jardin des Sciences, université de Strasbourg; la Mission patrimoine au sein de la Commission culture scientifique et technique, université de Rennes 1 ; la Mission à la Culture scientifique, université de Bourgogne ; le laboratoire d'étude et de conservation du patrimoine (partenariat muséum-université de Toulouse 3) ; le partenariat aquarium-université de Nancy...

(14) PATSTEC, www.patstec.fr/

(15) Trans Tyfipal, http://transtyfipal.u-bourgogne.fr/

(16) Par exemple, le quartier des Sciences à Toulouse ; la maison des Arts et des Sciences et conservatoire des collections à Strasbourg

(17) Enquête informelle et non publiée conduite en 2001 par Marta C. Lourenço.

(18) Voir http://museudaciencia.inwebonline.net/

(19) Au moment ou nous écrivons cet article, un jardin botanique universitaire est en danger : le jardin botanique de l'université libre d'Amsterdam, aux Pays-Bas, est menacé de fermeture pour permettre l'expansion du nouveau centre médical de l'université (Freek Vrugtman, dans litt. 24 March 2009).

(20) Pour une discussion plus approfondie de la problématique des musées et collections universitaires, voir Lourenço (2005).

(21) www.universeum.it/meetings.html

\section{Bibliographie}

Bergeron, A. Patrimoine et culture scientifique : sur l'inscription culturelle des savoirs, in Boudia S., Rasmussen, A. et Soubiran, S. (dir.), Patrimoine, savoirs et communautés savantes, Rennes : Presses universitaires de Rennes, (à paraître).

Boudia, S., Patrimoine et collections scientifiques des universités, U-culture, $\mathrm{n}^{\circ} 2,2007$, pp. 30-35.

Boudia, S., Rasmussen, A. et Soubiran, S. (dir.), Patrimoine, savoirs et communautés savantes, Rennes: Presses universitaires de Rennes, (à paraître).

Bremer, T. et Wegener, P. (dir.), Treasures of University Collections in Europe. Alligators and Astrolabes. Halle : Universität, 2001.
Choffel-Mailfert, J. Une politique culturelle à la rencontre d'un territoire : culture scientifique et industrielle en région Lorraine, 1980-1995. Paris : L'Harmattan, 1999.

Declaration of Halle, Academic Heritage and Universities - Responsibility and Public, 2000. www.universeum.it/declaration.html

Ferriot, D. et Lourenço, M.-C. De l'utilité des musées et collections des universités, la Lettre de l'OCIM, n93, 2004, pp. 4-16.

Gérard, P.-A. (dir.) Les collections scientifiques des universités. Nancy : Presses universitaires de Nancy, 2008.

Heesen, A. In medias res - Zur Bedeutung von Universitätssammlungen, NTM, n 16,2008 , pp. 485-490.

ICOM, Cahiers d'Études - Study Series, n¹1, 2003.

Lourenço, M.-C. Between two worlds: The distinct nature and contemporary significance of university museums and collections in Europe. Thèse de Doctorat, sous la direction de D. Ferriot, conservatoire national des Arts et Métiers, Paris, 2005 (http://webpages.fc.ul.pt/ mclourenco/).

Sanz, N. et Bergan, S. (dir.) Le patrimoine des universités européennes. Strasbourg : Conseil de l'Europe, 2002.

Stanbury, P. Adding value to university collections, Museologia, n³, 2003, pp. 1-4.

Such, M.-M. Associación de Museos y Collecciones Universitarios Españoles, Cahiers d'Études - Study Series, ICOM, n¹1, 2003, pp. 21-22.

Theologi-Gouti, P. Meeting of the Greek University Museums and Collections, UMAC Newsletter, janvier 2005, p. 4.

Université de Strasbourg, Gestion et mise en public des collections universitaires. Symposium organisé les 26 et 27 février 2009 à Strasbourg, vidéo accessible en ligne,www.canalc2.tv/

Université des Sciences et Technologies de Lille 1, Regards sur le patrimoine culturel des universités. Actes électroniques du séminaire national interministériel, $1^{\text {er }}$ et 2 avril 2004,

http://ustll.univ-lillel.fr/culture/agenda/04/patrimoine/patrimoine.html

Weber, C. Universitätssammlungen in Deutschland - Untersuchungen zu Bestand und Geschichte, in Burckhard, D., Hohls, R. et Prinz, C (dir.) Geschichte im Netz : Praxis, Chancen, Visionen. Teilband II : Historische Fachund Themenportale, Reihe Historisches Forum, 2007, pp. 228-243 (http://edoc.hu-berlin.de/histfor/10_II/).

Weber, C.et Lourenço, M.-C. UMAC Worldwide Database, in Tirrell, P.B.(dir.), Proceedings of the third conference of the International Committee for University Museums and Collections, UMAC, 2005, pp. 43-46. 